\title{
Information Sharing in a Long-term Supply Chain Relationship: The Role of Customer Review Strategy
}

\author{
Z. Justin Ren* Morris A. Cohen` Teck H. Ho; Christian Terwiesch ${ }^{\dagger}$
}

Revision: October 212007

\begin{abstract}
In this paper, we study the practice of forecast sharing and supply chain coordination with a game-theoretical model. We find that in a one-shot version of the game, forecasts are not shared truthfully by the customer. The supplier will rationally discount the forecast information in her capacity allocation. This results in Pareto sub-optimality for both supply chain parties. However, we show that a more efficient, truth-sharing outcome can emerge as an equilibrium from a long term relationship. In this equilibrium, forecast information is transmitted truthfully, and trusted by the supplier who in turn allocates the system-optimal equilibrium. This leaves both customer and supplier better off, compared to the non-truthful-sharing equilibrium.

We identify a multi-period review strategy profile that supports the truthful-sharing equilibrium. The key element of this strategy is that the supplier computes a scoring index of the customer's behavior that is updated over time and used to evaluate if the customer has sufficient incentive to share his private information truthfully in each transaction of the repeated game. Compared to trigger strategies, review strategies are more tolerant, but require diligence and more monitoring effort.
\end{abstract}

\footnotetext{
*Operations and Technology Management Department, Boston University School of Management. ${ }^{\dagger}$ Operations and Information Management Department, The Wharton School, Univ. of Pennsylvania. ${ }^{\ddagger}$ Marketing Group, Haas School of Business, University of California-Berkeley.
} 


\section{Introduction}

In November 2004, American Airlines announced that it was deferring the purchase of 54 out of 56 Boeing aircraft originally scheduled for delivery between 2006 and 2010. The financial impact for Boeing as the supplier was estimated to be as high as $\$ 2.7$ billion (Wall Street Journal, November 22, 2004). This example highlights the inherent problem in sharing forecast information in a supply chain. Forecasts provide information about what the buyer intends to do in a given future state of the world. These intentions (often expressed in the form of preliminary purchase orders or soft orders), however, cannot be easily verified or enforced in court.

To overcome the problems associated with forecast sharing, previous research has suggested a set of contracts that align the incentives in the supply chain and induce the buyer to reveal demand information truthfully. Such contracts include two-part tariff and three-part quadratic contracts (Desai and Srinivasan 1995, Corbett et al 2004), and capacity commitment and option contracts (Cachon and Lariviere 2001, Ozer and Wei 2006). Despite their theoretical appeal of creating a 'win-win' situation for buyer and supplier, empirical and anecdotal evidence shows that simple linear pricing contracts are still the most widely used format (Arrow 1985), possibly due to their simple structure and associated low administrative cost (Cachon and Lariviere 2005). For example, the aircraft industry (see Boeing example above), the semiconductor equipment industry (Cohen et al. 2003), and many other industries (Desai and Srinivasan 1995, Bajari and Tadelis 2001, Iyer and Villas-Boas 2003) still prefer the simple linear price contracts over the more complex coordinating contracts advocated by the supply chain literature. While one could discount this empirical evidence as irrelevant and simply declare Fortune 500 companies such as Boeing and Intel as laggards with respect to adopting the latest supply chain research, we offer an alternative explanation.

Most prior treatments of forecast sharing have used static or one-shot games. That is, in these models buyers and suppliers transact only once as in a spot-buy market. This eliminates any value of long-term relationships and thereby constrains the involved parties to ignore 
reputation. However in practice, many business relationships are long-term. Harmonious and mutually successful long-term supplier relationships have been observed in many industries and documented by researchers in strategy and management (e.g., McMillan 1990, Hagen and Choe 1998). In these supplier relationships, both the buyer and supplier are concerned about how their current strategic behavior affects their future interactions, and value longterm cooperation over one-shot business transactions. In fact, some buyers strongly prefer to deal with suppliers on a long-term basis, an observation that has been made repeatedly, especially in the automotive industry (Fujimoto 1999).

While multiple forms of contracts exist that can achieve credible information sharing, we find that having a repeated relationship itself can also induce truthful information sharing. A long-term relationship between supply chain parties gives each party opportunities to review the credibility of the other party, reward truth-telling, punish otherwise, and therefore provides the right incentive for truthful information sharing. In other words, repeated sourcing interactions can promote and form an implicit 'relational contract' among supply chain parties, which can be viewed as an alternative to complex pricing contracts in achieving supply chain coordination.

The present paper analyzes a forecast sharing game between one supplier and one customer under information asymmetry (The customer has superior information about demand compared to the supplier). There are potentially two sources of inefficiency in this supply chain setting. The first inefficiency results from "double marginalization" (Spengler 1950). It is well-know that the supplier operating with a linear wholesale contract would order less than the system-optimal quantity due to the difference between its profit margin and that of the supply chain. The other source of inefficiency results from information asymmetry. While previous literature has identified contracting mechanisms that are able to overcome both sources of inefficiency and achieve supply chain coordination (Cachon and Lariviere 2001; Ozer and Wei 2006), here we restrict the contracting space to the sole usage of linear price contracts (with no other instruments, such as fixed payments or advance purchase orders). 
We show that under certain conditions an efficient equilibrium can still emerge through the repeated supply chain relationship.

This paper makes the following contributions. First, we are - to the best of our knowledge - the first to establish that truthful information sharing can be achieved in an infinitely repeated forecasting game setting. Our repeated game model hence complements extant literature on supply chain contracting by introducing a long-term perspective (Pyke and Johnson 2003; Taylor and Plambeck 2006, 2007). Second, we expand the strategy space beyond the commonly-used trigger strategies and study review strategies in the context of forecast sharing. While trigger strategies act on signals received in one period, review strategies are based on signals collected over a number of periods. The usage of review strategies in studying repeated games with imperfect monitoring is not new, but we are the first to show the applicability of review strategies to a supply chain setting with asymmetric information. Previous applications of review strategies have relied on some unbiased current-period public signal to serve as a monitoring mechanism. However, in our setting the customer not only has private information about the potential market size, but also privately observes actual demand, neither of which the supplier has perfect knowledge of. In this setting, we show that review strategies can still induce truthful information sharing and supply chain coordination in the long run if we utilize signals from past periods efficiently.

The remainder of this paper is organized as follows. We survey the relevant literature in $\S 2$. After analyzing the one-shot forecasting game (§3), we turn our attention to the analysis of the repeated game $(\S 4)$ and show that with an incentive-compatible multi-period review strategy, truthful information sharing and supply chain coordination can be achieved. In $\S 5$, we discuss comparative static results that offer managerial insight as to when such a long-term relationship works best in promoting truthful information sharing. In $\S 6$, we conclude. 


\section{Literature Review}

In this section, we briefly review supply chain coordination literature in the following order: one-shot games under perfect information, one-shot games under information asymmetry, and infinitely repeated games.

Previous literature has focused on studying supply chain coordination as a one-shot game. When there is perfect and symmetric information, various types of contracts have been identified that can achieve system coordination. These include buy-back (Pasternack 1985), quantity-flexibility (Tsay 1999; Barnes-Schuster et al. 2002), sales-rebate (Taylor 2002), quantity-premium contract (Tomlin 2003) and revenue-sharing (Cachon and Lariviere 2005) contracts.

When there exists information asymmetry in the supply chain, it matters who offers the contract. Cachon and Lariviere (2001) formulate a capacity procurement game, where the customer, who has some private information about demand, offers a signalling contract to the supplier. In order to induce the supplier to provide enough capacity for high demand, a buyer truly expecting high demand has to use more than just a linear price contract to signal its type. For example, the customer can offer a fixed side payment to the supplier in order to separate himself from a low-demand type customer. While Cachon and Lariviere (2001) study such signalling contracting mechanisms in inducing truthful information sharing in a one-shot game, we use repeated games and investigate the effectiveness of just having a long-term relationship on truthful information sharing and supply chain coordination.

Screening contracts provide an alternative coordination mechanism. Porteus and Whang (1999) study capacity procurement with a screening model, where a supplier, who is unsure about the market size, offers the contract to a buyer who has the market information. Other screening models include Ha (2001) and Corbett et al. (2004). Ozer and Wei (2006) study

a capacity procurement game with asymmetric information similar to Cachon and Lariviere (2001), but they consider both signalling and screening contracts. When the manufacturer 
can signal her forecast information with an advance purchase, it leads to a signalling game. On the other hand, the less-informed supplier can induce the truthful forecast information from the manufacturer by offering a capacity reservation contract, which is a screening game. Moreover, Ozer and Wei (2006) show that channel coordination is possible when combining a capacity commitment contract with a pay-back contract. In this paper, we also consider channel coordination with information asymmetry. However, we use neither signalling nor screening devices. Instead, we resort to the repeated nature of supplier relationships, and show that coordination is achievable in the long-term even with a simple linear contract. Other papers that study incentives in information sharing include Lariviere and Padmanabhan (1997), Van Mieghem (1999), Corbett (2001), and Chen (2005). Chen (2002) provides an extensive literature review on information sharing and supply chain coordination.

During recent years, building a long-term supplier relationship has been increasingly recognized as an important aspect of supply chain management. Beth et al. (2003) pointed out that building a relationship is the key in managing supply chains, instead of investing in modern technologies. Pyke and Johnson (2003) study a spectrum of spot-buy and longterm sourcing relationships and identify the key drivers of each type of relationship. Taylor and Plambeck (2006) study a repeated supply chain relationship, in which production cost is uncertain and therefore ex ante binding contracting is infeasible. They identify an optimal 'relationship contract' that maximizes the customer's expected profits. They also demonstrate that better information visibility in monitoring unobserved actions can improve system performance significantly. We develop a qualitatively similar insight in this paper, but in a different setting. Another point of difference is that instead of allowing pre-game 'settlement' in each stage game, as done in Taylor and Plambeck (2006), we do not allow for this possibility. Instead we restrict the contracting space to linear transfer payments after capacity is built and demand is realized. Taylor and Plambeck (2007) further investigate two forms of relational contracts, where the buyer can either promise to pay a specific price, or can promise to purchase a specific quantity. These contractual arrangements are both easy to implement, 
and the authors show that which option is optimal to the buyer depends on the production and capacity cost, as well as the discount factor. In our model, the form of transfer payment, i.e., a linear contract, is pre-determined, and our focus is on achieving truthful forecast sharing in a long-term relationship.

In infinitely repeated games trigger strategies are the most commonly studied type of strategy (Friedman 1986), and are often adopted in the relational contracting literature (e.g., Baker et al. 2002, Levin 2003, Taylor and Plambeck 2003, 2006, Plambeck and Taylor 2007). In our setting it can be shown that trigger strategies can induce truthful forecast sharing but not system coordination, as punishment will be triggered in equilibrium due to the fact that the supplier cannot perfectly observe customer demand. Therefore we seek alternative strategies that can not only induce truthful information sharing but also system coordination. We find that using a properly defined review strategy can achieve this goal. Review strategies were first studied in economics in the context of repeated principal-agent model under imperfect monitoring. Radner (1985) studies repeated principal-agent games where the agent's action is not perfectly observed. He shows that by constructing a review strategy the repeated game can achieve asymptotic efficiency. We utilize a similar review strategy in this paper. However, the monitoring mechanism in our model is different. In Radner (1985), the outcomes from agent's private actions are observable, and can serve as a monitoring tool. In our setting, the realized demand sequences are only perfectly observed by the customer and cannot be directly used to evaluate the customer's truthfulness. Instead, the history of forecasts and orders by the customer are used to access the customer's truthfulness, which are publicly observable.

The game we study also belongs to a class of games called cheap-talk games (see, for example, Crawford and Sobel 1982). Cheap-talk games are different from signaling games in that the signals that are being sent in a cheap-talk game are costless, whereas the signals sent in signaling games must have cost implications (Gibbons 1992, page 210). When cheap-talk games are played repeatedly, it is generally unclear if efficient outcomes can be sustained. That is, the well-known folk theorem (Friedman 1971) may not apply in such games. For 
example, Morris (1998) considers two types of senders with different preferences over receiver's actions, and finds that truth-telling is not an equilibrium even in the infinitely repeated game. On the other hand, Stocken (2000) studies the credibility of a firm's manager disclosing private information to an outside investor, and shows that the manager does not have an incentive to truthfully reveal his information in a single-shot game, but can be motivated to do so if such a game is played repeatedly. Different from the above mentioned papers, this paper has an additional source of information asymmetry in the public signal received after actions are taken. The customer not only privately observes the demand size parameter, he also privately observes the true demand realization, neither of which the supplier observes perfectly.

\section{The Model: One-shot Game}

In the supply chain we study, there is one customer (also called the "buyer", referred to as 'he') and one supplier (referred to as 'she'). The customer wants to acquire a certain amount of capacity from the supplier before actual demand is realized. We assume that the market demand the customer faces is a scaled random variable $\theta \cdot X$, where $X$ is non-negative normal random variable with mean $\mu$ and standard deviation $\sigma$. Define $\theta \in \Re^{+}$as the demand size parameter. To capture the essence of the uncertain market condition, we assume that $\theta$ is a random variable with two possible values "high" and "low": $\theta_{i}, i=\{h, l\}$. The probability of each happening is $P\left(\theta_{l}\right)=\alpha, P\left(\theta_{h}\right)=1-\alpha, \alpha \in(0,1)$. The high and low market demand random variables are therefore $D_{h}=\theta_{h} X$, and $D_{l}=\theta_{l} X$, respectively. Denote their CDF $F_{h}($.$) and F_{l}($.$) , respectively. It is assumed that \theta$ is independent of $X$. The distributions of both $\theta$ and $X$ are common knowledge.

The supplier's unit capacity cost is $c$, and she charges the customer a price of $r$ for each unit allocated and utilized. The customer earns a unit revenue of $p$ for each unit of capacity allocated and utilized. When the capacity allocated is not enough to satisfy realized demand, the customer incurs a unit cost of $g$. This includes potential revenue lost, loss of goodwill, or 
the additional cost to find alternative sources of supply. On the other hand, if the supplier allocates too much capacity, for those units not purchased by the customer, a unit overage cost of $h$ is incurred. All the cost parameters are public knowledge. For notational convenience, define

$$
\begin{aligned}
& u\left(\theta_{i}, K\right)=r \min \left(K, D_{i}\right)-h\left(K-D_{i}\right)^{+}-c K \\
& v\left(\theta_{i}, K\right)=(p-r) \min \left(K, D_{i}\right)-g\left(D_{i}-K\right)^{+}
\end{aligned}
$$

Where $(x)^{+}=\max (x, 0)$.

The game begins with a random draw of $\theta$ by the customer. The customer observes this realization of $\theta$ (either $\theta_{h}$ or $\theta_{l}$ ), but the supplier does not. The customer then sends a nonbinding forecast $m$ to the supplier. Here we consider the simplest forecast information set: either $m=H$, or $m=L$. The supplier's strategy is also binary: to trust, or not to trust the customer's forecast. If the supplier trusts the customer's forecast, then she builds capacity according to the received forecast. That is, if she receives a forecast of $H$ from the customer and she trusts the customer, then she would allocate capacity $K_{h}$ that maximizes her expected profit believing that the market demand random variable would be $D_{h}$ :

$$
K_{h}=\arg \max _{K} \mathrm{E}\left[u\left(\theta_{h}, K\right)\right]
$$

Similarly, if the supplier trusts the customer and receives a forecast of $L$, then she would allocate capacity $K_{l}$ that maximizes her expected profit believing that the market demand random variable would be $D_{l}^{\vdots}$ :

$$
K_{l}=\arg \max _{K} \mathrm{E}\left[u\left(\theta_{l}, K\right)\right]
$$

On the other hand, if the supplier does not trust the customer, then his forecast is considered meaningless. The supplier then allocates capacity $K_{0}$ as if there were no meaningful forecast being shared at all:

$$
K_{0}=\arg \max _{K}\left\{(1-\alpha) \mathrm{E}\left[u\left(\theta_{h}, K\right)\right]+\alpha \mathrm{E}\left[u\left(\theta_{l}, K\right)\right]\right\}
$$


We assume that capacity is publicly observable to the customer. This may not be true in every situation (see Taylor and Plambeck 2006 for a model with unobservable capacity choices), but is reasonable for industries where production volume can easily be observed. For example, we found in our prior research in the semi-conductor equipment supply chain as well as in the aerospace and defense sector that customers often have on-site personnel (often to support on site quality assurance and testing) who have detailed knowledge about current production volumes. After capacity is installed, the true market demand (i.e., a draw from the underlying distribution of $\theta X)$ is realized, which we label as $d$. The customer places an order $o$. It is easy to see that in the one-shot game, the customer has no incentive to order anything other than the true demand.

In the truthful information sharing situation (the customer shares his forecast truthfully and places his order equal to true demand and the supplier trusts the forecast), the customer's expected profit $v^{*}$ and the supplier's expected profit $u^{*}$ are:

$$
\begin{aligned}
& v^{*}=(1-\alpha) \mathrm{E}\left[v\left(\theta_{h}, K_{h}\right)\right]+\alpha \mathrm{E}\left[v\left(\theta_{l}, K_{l}\right)\right] \\
& u^{*}=(1-\alpha) \mathrm{E}\left[u\left(\theta_{h}, K_{h}\right)\right]+\alpha \mathrm{E}\left[u\left(\theta_{h}, K_{h}\right)\right] .
\end{aligned}
$$

These expected payoffs correspond to the cooperative case. In the non-cooperative case, the supplier ignores the customer's forecasts and allocates capacity according to her prior knowledge about $\theta$ alone. The expected profits are:

$$
\begin{aligned}
& v^{0}=(1-\alpha) \mathrm{E}\left[v\left(\theta_{h}, K_{0}\right)\right]+\alpha \mathrm{E}\left[v\left(\theta_{l}, K_{0}\right)\right], \\
& u^{0}=(1-\alpha) \mathrm{E}\left[u\left(\theta_{h}, K_{0}\right)\right]+\alpha \mathrm{E}\left[u\left(\theta_{h}, K_{0}\right)\right] .
\end{aligned}
$$

Table 1 summarizes the notation used in the one-shot game.

The following characterizes the equilibrium of the one-shot game: (its proof is provided in the Appendix)

Proposition 1 In the equilibrium of the one-shot game, the supplier ignores the customer's forecast information, and allocates capacity $K_{0}$ regardless of demand state $\theta$. 


\begin{tabular}{ll}
\hline$\theta_{i}, i=\{h, l\} ; \alpha$ & demand size parameter; probability of low market demand $\left(\theta_{l}\right)$ \\
$p, r$ & customer's unit revenue, and transfer price to the supplier for capacity allocated and utilized \\
$c, h, g$ & supplier's unit capacity cost, overage cost, and shortage cost \\
$D_{i} ; F_{i}() ; d$. & end-market demand the customer faces; CDF for $D_{i} ;$ actual demand realization from $D_{i}$ \\
$m ; o$ & customer's forecast; customer's order after observing demand \\
$K_{i} ; v^{*}, u^{*}$ & capacity under cooperation; and the resulting expected profit for customer and supplier \\
$K_{0}, v^{0}, u^{0}$ & capacity under non-cooperation; and the resulting expected profit for customer and supplier
\end{tabular}

Table 1: Notation: One-shot game

Driving the above results is the customer's underlying incentive to overforecast. While the customer with a high demand wants to reveal the truth to the supplier, the low-demand customer also wants to send a high-demand forecast so that he could have more capacity allocated to him, i.e., he would like to mimic a high-demand customer. Expecting such an incentive to overforecast from the customer, the supplier cannot distinguish the true demand state, and is better off ignoring the customer's forecasts. Such a reaction from the supplier results in a non-truthful forecast-sharing equilibrium. A similar result is obtained in Ozer and Wei (2006). After demand is realized, though, the customer always places his order according to true demand $(o=d)$, because ordering any other amount has no benefit, and can only hurt his expected profit.

This equilibrium is inefficient. It is easy to see that there exist a set of capacity levels $K_{i}^{\prime}$ that pareto-dominates $K_{0}$ :

$$
\begin{aligned}
& (1-\alpha) \mathrm{E}\left[v\left(\theta_{h}, K_{h}^{\prime}\right)\right]+\alpha \mathrm{E}\left[v\left(\theta_{l}, K_{l}^{\prime}\right)\right] \geq v^{0}, \\
& (1-\alpha) \mathrm{E}\left[u\left(\theta_{h}, K_{h}^{\prime}\right)\right]+\alpha \operatorname{E}\left[u\left(\theta_{l}, K_{l}^{\prime}\right)\right] \geq u^{0} .
\end{aligned}
$$

The capacity levels under the cooperative solution as defined in eq. (3) and (4) belong to this set. Another focal candidate is the supply-chain optimal capacity levels $K_{i}^{c}$ :

$$
K_{i}^{c}=\arg \max _{K} \mathrm{E}\left[v\left(\theta_{i}, K\right)+u\left(\theta_{i}, K\right)\right]
$$


Unfortunately in the one-shot game, none of the those pareto-dominant capacity levels can happen in equilibrium because of the misaligned incentives induced by information asymmetry and by double marginalization. A natural question is therefore to ask under what circumstances the more desirable outcomes can emerge. In the next section, we investigate the forecast sharing game in the context of a long-term supply chain relationship.

\section{A Long-term Supply Chain Relationship}

We model a long term supplier relationship as a repeated game between customer and supplier. The repeated game consists of the following features. Let time be indexed by $t=1,2, \ldots, \infty$. At each time $t$, a stage game is played, starting with a random draw of $\theta$, which we denote as

$\theta_{t}$. The stage game is identical to the one-shot game: (1) After privately observing $\theta_{t}$, the customer's action is to choose which forecast to provide to the supplier, denoted by $m_{t}$. (2) The supplier's action is to install a capacity level $K_{t}$. (3) After capacity $K_{t}$ has been allocated, true demand $d_{t}$ is realized. (4) The customer then places the order of $o_{t}$ to fulfill demand. If $K_{t}<o_{t}$, only $K_{t}$ units of the orders are satisfied, and the remaining unsatisfied orders are lost. If $K_{t} \geq o_{t}$, then the entire order is satisfied. (5) The supplier is paid according to a linear pricing contract with a unit rate of $r$. This concludes the one stage game. Then time is advanced to $t+1$, and the game renews, with another independent draw of $\theta_{t+1}$.

The public information at the start of period $t$ consists of all the past forecasts shared $m^{t}=$ $\left(m_{1}, \ldots, m_{t-1}\right)$, orders placed $o^{t}=\left(o_{1}, \ldots o_{t-1}\right)$, and capacity levels installed $K^{t}=\left\{K_{1}, \ldots K_{t-1}\right\}$. Collectively, this information is called the public history at time $t: h^{t}=m^{t} \times o^{t} \times K^{t}$, which is fully observed by both parties. In contrast, the private history of the repeated game consists of all the demand signals and demand realizations that the customer privately observed: $h_{\text {private }}^{t}=\left(\theta_{1}, \ldots, \theta_{t-1}\right) \times\left(d_{1}, \ldots, d_{t-1}\right)$.

The customer chooses a strategy corresponding to a sequence of functions that map the public history and his private information into forecasts and orders: $h^{t} \times \theta_{t} \rightarrow m_{t}, o_{t}, \forall t$. The 
supplier chooses a strategy corresponding to a sequence of functions that maps the public history into capacity decisions: $h^{t} \rightarrow K_{t}$. Note that neither party's strategies depend on private history $h_{\text {private }}^{t}$, hence they are public strategies (Fudenberg and Tirole 1991).

Let the discount rate be $\delta_{0}$. To model an uncertain lifetime of the customer-supplier relationship, suppose at the end of each period, there is a probability $p_{0}$ that relationship will be terminated. Hence $\delta=\delta_{0}\left(1-p_{0}\right)$ is the effective discount factor.

A perfect public equilibrium (PPE) is a profile of public strategies that starting from any period $t$, given any public history $h^{t}$, constitutes a Nash equilibrium from that point onward (Osborne and Rubinstein 1994).

Below we show that a pareto-efficient outcome can emerge as an equilibrium in the repeated game using a strategy profile called a multi-period review strategy. This is accomplished by the supplier setting up a scoring system and evaluating the customer's credibility over a number of consecutive periods. Review strategies have previously been studied in the economics literature (Radner 1985, Stocken 2000). In contrast to the widely-studied trigger strategies, a review strategy in the context of a repeated game with imperfect monitoring does not prescribe immediate punishment once the customer fails the supplier's credibility test in one period. Instead, the customer is given more chances over a longer period of time. As a result, the likelihood of mistakenly punishing a truth-telling player is smaller. On the other hand, the review strategy needs to be stringent enough so that the customer has no incentive to 'coast' once he accumulates high enough credits. Therefore the parameters of the review strategy, particularly the review length and the threshold for punishment need to be set carefully. Below we explicitly derive a review strategy that achieves both truth-telling and improves efficiency for both parties.

Our review strategy works as follows. Time is divided into a sequence of review phases, each having a maximum length of $R$ periods. During each review phase, the customer reveals information truthfully, and the supplier maintains an index (i.e., a 'scorecard') $I_{t}$ and a credibility threshold $\gamma_{t}$. At the end of each period $t \in[1, R]$, after order $o_{t}$ is placed, the supplier 
evaluates the truthfulness of the customer with a credibility test, and updates the score $I_{t}$.

If the customer reports demand to be high $\left(m_{t}=H\right)$, the customer's score is subject to the following two tests. The first test evaluates the proportion of periods in which the customer reported high demand $\left(M_{t}=H\right)$ so far in the review period. Because in each period the high demand scenario happens with probability $1-\alpha$, in the long run the supplier should observe approximately $1-\alpha$ proportion of the forecasts be high demand, assuming the customer reports $\theta_{t}$ in each period truthfully. Denote $N_{t}$ the number of periods up to $t$ in a review phase in which the customer reports high demand. The sampling distribution of $\alpha$ is approximately normal with mean $\alpha$ and standard deviation $\sqrt{\alpha(1-\alpha) /\left(t-N_{t}\right)}$. Let $z_{\alpha}$ be the value associated with a confidence level $p_{\alpha}$ (for example $z_{a}=1.96$ for a confidence level of $p_{\alpha}=95 \%$ ). The customer is then judged to pass the first test in period $t$ if the proportion of high demand reported up to $t$ is no greater than $(1-\alpha)+z_{a} \sqrt{\alpha(1-\alpha) / N_{t}}$. This test is designed to reduce the customer's incentive to excessively over-forecast and report high demand, as is the case in the one-shot game.

The second test analyzes the actual order quantities $o_{t}$. A threshold $d_{h, t}$ is set according to the following statistical test. If the customer is reporting truthfully, then the sample mean of the demand realizations from those high demand periods $\left(\theta_{h}\right)$ is normally distributed with mean $\theta_{h} \mu$ and standard deviation $\sigma \sqrt{\theta_{h} / N_{t}}$. Let $z_{h}$ and $p_{h}$ be the critical value and probability associated with a certain significance level (for example, $z_{h}=1.96$ for a significance level of $\left.p_{h}=95 \%\right)$. Then $\underline{d_{h, t}}=\theta_{h} \mu-z_{h} \sigma \sqrt{\theta_{h} / N_{t}}$. The customer passes the second test if the average order size in those reported high demand periods up to time $t$ exceeds $d_{h, t}$. Only after the customer passes both of the above tests can his score be incremented, $I_{t}=I_{t-1}+1$.

If the customer reports demand to be low $\left(m_{t}=L\right)$, the customer is evaluated with only one test related to the actual order he places. If the customer is ordering truthfully, the sample mean of the demand realizations from those low demand periods is normally distributed with mean $\theta_{l} \mu$ and standard deviation $\sqrt{\theta_{l}} \sigma$. Let $z_{l}$ and $p_{l}$ be the critical value and probability associated with a certain significance level (for example, $z_{d}=1.96$ for a significance level of 
$\left.p_{l}=95 \%\right)$. Then $\overline{d_{l . t}}=\theta_{l} \mu+z_{l} \sigma \sqrt{\theta_{l} /\left(t-N_{t}\right)}$. The customer passes the test if his average order size in the reported low demand periods in the review phase up to $t$ is no greater than $\overline{d_{l . t}}$. If this happens, then his score is incremented by one, $I_{t}=I_{t-1}+1$. On the other hand, if he fails either test, his score is not updated, $I_{t}=I_{t-1}$.

The overall probability that the customer is evaluated as truthful is $S=(1-\alpha) p_{h} p_{\alpha}+\alpha p_{l}$. This measures the strength of the credibility checking system. The credibility threshold $\gamma_{t}$ is defined as $\gamma_{t}=\frac{R S-J}{R} t$, where $J$ is some allowance for measurement error. When $J=0$, the credibility threshold is simply $\gamma_{t}=S t$.

At the end of each period, the supplier also evaluates whether the customer still has an incentive for truth-telling. If he does, the review phase is continued. If the supplier finds that the customer no longer has an incentive to report forecasts truthfully, the review phase is terminated. Upon termination of the review phase, if the supplier score $I_{t} \geq \gamma_{t}$, the customer passes the review and the games restarts with a new review phase. If $I_{t}<\gamma_{t}$, the customer fails the review and the games enters a non-truthful-sharing (punishment) phase that lasts for $M=\chi R$ periods, where $\chi>0$.

During the review phase, the supplier always trusts the customer, and builds systemoptimal capacity according to the customer's forecasts of market demand. During the punishment phase, the supplier ignores the customer's forecasts, and the customer does not share forecasts truthfully. Therefore the game reverts to the one-shot uncommunicative Nash equilibrium for $M$ periods. The repeated game begins with a review phase, with supplier score $I_{0}=0$. Let the customer's total discounted expected payoff at the beginning of the game be denoted as $V\left(I_{0}\right)$, and let the customer's one-stage payoff associated with the supply-chain optimal capacity level $K_{i}^{c}$ (as defined in equation 10) be denoted as $v^{c}$, i.e., $v^{c}=(1-\alpha) \mathrm{E}\left[v\left(\theta_{h}, K_{h}^{c}\right)\right]+\alpha \mathrm{E}\left[v\left(\theta_{l}, K_{l}^{c}\right)\right]$.

Theorem 2 For all $\varepsilon>0$, there exists a discount rate $\underline{\delta}$, a review phase of length $\underline{R}$, and a credibility assessment value $\underline{S}$ such that for $\forall \delta>\underline{\delta}, R>\underline{R}, S>\underline{S}$ there exists a perfect public equilibrium of the repeated game in which $V\left(I_{0}\right)>v^{c}-\varepsilon$, provided that the efficient capacity 
levels $K_{i}^{c}$ yield both parties no worse off than their expected profits with no forecast sharing. In this equilibrium, the customer always shares demand information as well as places orders truthfully, and the supplier allocates the system-optimal capacity $K_{i}^{c}$.

The proof for this Theorem is provided in the Appendix.

The above theorem not only stipulates that there exist parameters of the game that can support a truthful-information-sharing equilibrium, it also states that in such an equilibrium the non-truthful-information-sharing punishment phase would almost never be observed even in the presence of asymmetric information. This is because with a review strategy such as defined above, the customer is given an extended period of time to prove his intention of sharing information truthfully. If, for example, the customer had bad luck and had a very low demand realization in one period but had forecasted high demand in good faith, the customer would not be punished immediately. Instead, his credibility score would suffer but he would still be regarded as trust-worthy and be given more periods to vindicate himself. On the other hand, if the customer intentionally over-forecasted demand, he may not be caught immediately, but the credibility tests are designed such as repeated lying would be caught. This is feasible because the supplier has information on the distribution of both demand size parameters, as well as those of each demand distributions. Therefore hypothesis tests on sample means can be utilized with repeated samples. In the long run, the review strategy ensures that the probability that a truth-telling customer fails the review goes to zero as the number of review periods $R$ becomes large (provided also that the discount factor $\delta$ is sufficiently high, and the credibility check system $S$ is effective enough). Also note that as $R$ becomes large, the length of the punishment phase is also longer. This also increases the customer's incentive to share forecast information truthfully. The effect of the inefficiency brought by the longer punishment phase is mitigated by a reduction in the probability of inappropriately punishing a customer who has been sharing forecast information truthfully. As is shown, for a sufficiently long review period, the probability of the customer failing the review when sharing his forecasts truthfully can be bounded arbitrarily close to zero. Hence, 
the supply chain converges to a fully efficient coordinated equilibrium.

\section{Discussion}

In this section, we provide a set of managerial implications of our findings. Specifically, we study how credibility test, time horizon, length of the punishment phase, and the discount factor will make truthful forecast sharing more or less likely.

First, consider how the credibility test works in detecting if a customer is truthful in sharing his demand forecasts. Because the supplier cannot observe true demand quantities directly, she has to rely on past demand information. In order to make sure that the customer does not always over-forecast, the supplier can look at how often the customer has forecasted high demands and then determine if the proportion of high demand is suspiciously high. This is analogous to the hypothesis testing used for the mean of sample proportions. Note that this credibility test is not memoryless. His past order quantities in his reported high (and low) demand periods are recorded. The supplier uses one additional statistical test on the sample mean of order quantities to see if they are indeed from a high (and low) demand distribution. This further reduces the customer's incentive to lie about actual demand.

The review strategy is constructed such that even if the customer is truthful and fails the test in one period, he is not being punished immediately. With a review-strategy, the customer is given an extended period of time to prove his intention of sharing information truthfully. Also, as the review length $R$ is extended, so is the the length of punishment phase. This increases the customer's incentive to share forecast information truthfully. A prolonged punishment phase, triggered by errors that are inherent in the credibility system under imperfect monitoring, is not pareto-efficient. However, such inefficiency is mitigated by a reduction in the probability of inappropriately punishing the customer who has been sharing forecast information truthfully.

Also, as the discount factor $\delta$ increases, future long-term gains from sharing the forecast 
truthfully, as well as the punishment threat from doing otherwise, become more important. Therefore the customer is less inclined to behave myopically and opportunistically. There are a number of factors that affect the discount factor $\delta$. For example, recall that $\delta=\delta_{0}\left(1-p_{0}\right)$ is a decreasing function of $p_{0}$, the probability that the repeated game may stop at the end of each stage game. Some industries are highly risky, with firms entering and exiting at a fast rate. In such industries, $p_{0}$ is high, and therefore firms tend to focus more on near-term payoff. As a result they tend to ignore the value of long-term relationships. In such environments, our model suggests that truthful information sharing is more difficult to obtain, and a long-term relationship is less likely to emerge.

Review strategies with long review periods $R$ can be observed in practice. For example, Sun Microsystems rates each of its suppliers' performance with a 'scorecard' system, and reviews the scores with the supplier each quarter. Suppliers are given multiple opportunities to meet the company's requirements. The review system helps Sun to achieve its goal of building long-term relationship with suppliers (Farlow et al. 1996). Companies such as Intel (Minahan 1998a), Chrysler (Minahan 1998b), and Toyota and Mitsubishi (Hagen and Choe 1998) all have similar review practices. Although in those examples scorecards are used to evaluate suppliers, our model suggests that such review systems can also be effective in gauging the customer's truthfulness in information sharing when some information cannot be symmetrically observed.

\section{Conclusion}

In this paper, we study the practice of forecast sharing and supply chain coordination with a game-theoretical model. We find that in a one-shot version of the game, forecasts are not shared truthfully by the customer. The supplier will rationally discount the forecast information in her capacity allocation. This results in Pareto sub-optimality for both supply chain parties. However, we show that a more efficient, truth-sharing outcome can emerge 
as an equilibrium from a long term relationship. In this equilibrium, forecast information is transmitted truthfully, and trusted by the supplier who in turn allocates the system-optimal equilibrium. This leaves both customer and supplier better off, compared to the non-truthfulsharing equilibrium.

We identify a multi-period review strategy profile that supports the truthful-sharing equilibrium. The key element of this strategy is that the supplier computes a scoring index of the customer's behavior that is updated over time and used to evaluate if the customer has sufficient incentive to share his private information truthfully in each transaction of the repeated game. Compared to single-period trigger strategies, multi-period review strategies are more tolerant, but require diligence and more monitoring effort. This is also consistent with what we observe in a coordinated supply chain. For example, Toyota when working with its suppliers, does not immediately sever the business relationship with any of its suppliers when it has quality issues or delivery problems, which fail to meet Toyota's standards. Instead, the company dispatches its own staff to the supplier's site and works with their suppliers for extended periods of time to help them correct the problem. Only under extreme circumstances does Toyota drop any supplier from its supplier base (Liker 2004). A similar observation is made in the semiconductor equipment industry (Cohen et al. 2003).

The implication of our findings is that when supply chain parties have a long-term ongoing relationship, or expect to do so, they can achieve coordination even though incentives are not aligned in the short run. In the case of forecast sharing, the customer has an incentive to overforecast if the supplier relationship is of short term nature. However, when the parties interact repeatedly, the threats of retaliation in response to bad behavior and the promise of cooperation in response to good behavior in the current period can have significant impact on future payoffs. Concerns about credibility and payoff in the long run can thus yield cooperation and coordination. This 'relational contract' is desirable especially when actions are not perfectly observable and cannot be contracted or enforced.

Our model has its limitations. There are only two demand states $\left(\theta_{h}\right.$ and $\left.\theta_{l}\right)$ in our model. 
An extension to multiple states will be more complex, as the tool for distinguishing each state from multiple potential states has to be more sophisticated. One can also generalize other aspects of the model. For example, even though in many supply chains capacity can be easily verified, in many others the supplier's capacity choice can be not perfectly observable or verifiable. As a result, the customer has to use some monitoring mechanism for detecting if the supplier has allocated the right amount of capacity. Incorporating this would enrich the model in a meaningful way. Currently in our model the demand state is drawn independently each period. It may be more realistic to consider a repeated model setting where demand states are correlated over time, and see how that affects the equilibrium. Alternatively, one can model the customer's type to be fixed over time (e.g., a honest type who always tells the truth, or a strategic type), and the supplier has to learn the customer's type over some periods of time. It would also be interesting to generalize the supply chain structure to one with one supplier and multiple customers, and see how the dynamics change in a long-term relationship, especially how competition among customers affects the equilibrium outcome. We hope to address these modeling issues in our future research.

\section{Acknowledgement}

The authors thank the Associate Editor and two anonymous reviewers for their helpful comments that much improved the paper. The authors also thank Gerard Cachon, Jiri Chod, Noah Gans, Serguei Netessine, Fuqiang Zhang and seminar participants at the Wharton School and MIT Sloan reading group for their helpful comments. Steve Graves and Yong-Pin Zhou provided detailed comments to an early version of the paper. This paper has benefited from helpful discussions with Phillip Stocken. This research was partially funded by two National Science Foundation grants (DMI01-21395 and DMI-0075391).

\section{Appendix: Proofs}


Proof. Proof of Proposition 1: Suppose the supplier trusts the customer and always reserves capacity according to his forecast. The high-type customer $\left(\theta=\theta_{h}\right)$ will sure send $H$ to reserve more capacity. However, the low-type customer also has an incentive to forecast $H$ even though he receives low demand $\theta=\theta_{l}$, because his profit is strictly increasing in capacity reserved. The supplier's expected profit would be higher if she just build $K_{0} \in\left[K_{l}, K_{h}\right]$, because

$$
\begin{aligned}
& (1-\alpha) \mathrm{E}\left[(p-r) \min \left(K_{h}, D_{h}\right)-g\left(D_{h}-K_{h}\right)^{+}\right]+\alpha \mathrm{E}\left[(p-r) \min \left(K_{h}, D_{l}\right)-g\left(D_{l}-K_{h}\right)^{+}\right] \\
& \leq(1-\alpha) \mathrm{E}\left[(p-r) \min \left(K_{0}, D_{h}\right)-g\left(D_{h}-K_{0}\right)^{+}\right]+\alpha \mathrm{E}\left[(p-r) \min \left(K_{0}, D_{l}\right)-g\left(D_{l}-K_{0}\right)^{+}\right]
\end{aligned}
$$

by the definition of $K_{0}$ (see equation 5 ). Therefore, trusting the customer's forecast is not a best response for the supplier. Given that the supplier's best response is to ignore the forecasts, then it does not matter what the customer forecasts, because his assigned capacity level is fixed at $K_{0}$. After demand is realized, the customer always placed its order according to the true demand realization, because ordering any other amount has no benefit, and can only hurt his own expected profit.

The following four lemmas are necessary to prove the main theorem (Theorem 2). But first we define the following:

$v\left(I_{0}\right)$ is the customer's normalized discounted expected profit at the beginning of the game;

$v\left(I_{R-n}^{x}\right)$ is the customer's normalized discounted expected profit when there are $n$ periods remaining in the review phase and the value of the trust index at period $t=R-n$ is $x$;

$q=R . S-J$, where $J$ is some allowable margin of error (whose exact value is to be defined in the latter part of the proof);

Lemma 1 Suppose the customer is truthful-telling always. For all $n$ such that $1 \leq n \leq q$,

$$
\begin{aligned}
v\left(I_{R-n}^{q-n}\right) & =(1-\delta) v^{c} \sum_{i=0}^{n-1}(S \delta)^{i}+v\left(I_{0}\right)(S \delta)^{n} \\
& +\left[\left(1-\delta^{M}\right) v^{0}+\delta M v\left(I_{0}\right)\right] \sum_{i=1}^{n}(1-S) \delta^{i} S^{i-1}
\end{aligned}
$$

Proof. By induction. When $n=1$, the customer either passes the review or the supply chain enter the 
punishment phase at the end of the period; Therefore

$$
\begin{aligned}
v\left(I_{R-1}^{q-1}\right) & =(1-\delta) v^{c}+\delta S v\left(I_{0}\right) \\
& +\delta(1-S)\left[\left(1-\delta^{M}\right) v^{0}+\delta^{M} v\left(I_{0}\right)\right] .
\end{aligned}
$$

Suppose the induction hypothesis holds for $n$.

$$
\begin{aligned}
v\left(I_{R-(n+1)}^{q-(n+1)}\right) & =(1-\delta) v^{c}+\delta S v\left(I_{R-n}^{q-n}\right) \\
& +\delta(1-S)\left[\left(1-\delta^{M}\right) v^{0}+\delta^{M} v\left(I_{0}\right)\right]
\end{aligned}
$$

Now we show that it is true for $n+1$. Substituting in the induction hypothesis yields:

$$
\begin{aligned}
& v\left(I_{R-(n+1)}^{q-(n+1)}\right) \\
& =(1-\delta) v^{c}+\delta S\left\{(1-\delta) v^{c} \sum_{i=0}^{n-1}(S \delta)^{i}+v\left(I_{0}\right)(S \delta)^{n}+\right. \\
& \left.\left(\left(1-\delta^{M}\right) v^{0}+\delta^{M} v\left(I_{0}\right)\right) \sum_{i=1}^{n}(1-S) \delta^{i} S^{i-1}\right\}+\delta(1-S)\left[\left(1-\delta^{M}\right) v^{0}+\delta^{M} v\left(I_{0}\right)\right] \\
& =(1-\delta) v^{c} \sum_{i=0}^{n-1}(S \delta)^{i}+v\left(I_{0}\right)(S \delta)^{n+1}+\left[\left(1-\delta^{M}\right) v^{0}+\delta^{M} v\left(I_{0}\right)\right] \sum_{i=1}^{n+1}(1-S) \delta^{i} S^{i-1} .
\end{aligned}
$$

Lemma 2 The customer will only fail the review when $I_{t}<\max (0, t-(R-q))$, i.e. when $I_{t}$ is such that the customer will fail the review with probability 1.

Proof. To show that the customer will truthfully share his private information when $I_{t}$ is such that $I_{t} \geq \max (0, t-(R-q))$, it is sufficient to show that the customer has an incentive to release his private information truthfully when $I_{R-n}=q-n$. This result is established in the following claim.

Claim: Let $I_{R-n}=q-n$ and consider $n$ such that $1 \leq n \leq q$. There exists $\underline{\delta}^{\prime}$ such that for all $\delta \in\left[\underline{\delta}^{\prime}, 1\right)$, there exists a $\underline{S}$ and $\underline{R}^{\prime}$ such that for all $S>\underline{S}$ and $R>\underline{R}^{\prime}$ the customer truthfully reveals his private information.

Proof: Consider a one-shot deviation when the customer observes $\theta_{l}$. Define the following: 
$\beta$ is the conditional probability that the supplier will assess the customer's truthful forecast of $\theta=\theta_{l}$ and orders to be truthful;

$\eta$ is the conditional probability that the supplier will assess the customer's behavior to be truthful when the customer observes $\theta=\theta_{l}$ and follows an optimal deviation strategy.

$v^{\prime}\left(\theta_{l}\right)$ is the expected one-stage payoff when the customer observes $\theta_{l}$ and follows his optimal deviation;

$$
\Delta=v^{\prime}\left(\theta_{l}\right)-v\left(\theta_{l}, K_{l}^{c}\right)
$$

$\lambda$ denotes the probability of failing the review conditional on being at the beginning of the game;

Note that the significance level for the credibility test can be chosen such that $\beta$ is close to 1 . On the other hand, $\eta$ is strictly less than 1 . Suppose not. Then the customer will order in some periods order quantities other than true demand in order to pass the test. Doing so will incur additional cost, as in this case of overordering, $r\left(o_{t}-d_{t}\right)^{+}$is wasted, whereas in the case of under-ordering a potential profit of $(p-r)(d-o)^{+}$ is lost. Moreover, the benefit of doing so decreases as $R$ increases, which we show below.

For any $R, I_{R-n}=q-n$ and any $n$ such that $1 \leq n \leq q$, the customer would share behave truthfully if:

$$
\begin{aligned}
& (1-\delta) v^{\prime}\left(\theta_{l}\right)+\delta \eta v\left(I_{R-(n-1)}=q-(n-1)\right)+(1-\eta)\left[\delta\left(1-\delta^{M}\right) v^{0}+\delta^{M+1} v\left(I_{0}\right)\right] \\
& \leq(1-\delta) v\left(\theta_{l}, K_{l}^{c}\right)+\delta \beta v\left(I_{R-(n-1)}=q-(n-1)\right)+(1-\beta)\left[\delta\left(1-\delta^{M}\right) v^{0}+\delta^{M+1} v\left(I_{0}\right)\right] .
\end{aligned}
$$

Rearranging terms, we have

$$
\Delta \leq \frac{(\beta-\eta)}{1-\delta} \delta\left[v\left(I_{R-(n-1)}=q-(n-1)\right)-\left(\left(1-\delta^{M}\right) v^{0}+\delta^{M} v\left(I_{0}\right)\right)\right]
$$

Using Lemma 1, which gives the customer's expected profit when $I_{R-n}=q-n$ for all $1 \leq n \leq q$, and substituting for $v\left(I_{R-(n-1)}=q-(n-1)\right)$ yields the condition:

$$
\begin{aligned}
\frac{(\beta-\eta)}{1-\delta} \delta\left\{(1-\delta) v^{c} \sum_{i=0}^{n-2}(S \delta)^{i}\right. & +v\left(I_{0}\right)(S \delta)^{n-1} \\
& \left.+\left(\left(1-\delta^{M}\right) v^{0}+\delta^{M} v\left(I_{0}\right)\right)\left(\sum_{i=1}^{n-1}(1-S) \delta^{i} S^{i-1}-1\right)\right\} \geq \Delta .
\end{aligned}
$$


Using L'Hospital's rule, we have as $\delta \rightarrow 1$,

$$
\begin{aligned}
& \frac{(\beta-\eta)}{-1}\left\{v\left(I_{0}\right) S^{n-1}+v\left(I_{0}\right)\left(\sum_{i=1}^{n-1}(1-S) S^{i-1}-1\right)\right. \\
& \left.-v^{c} \sum_{i=0}^{n-2} S^{i}+(n-1) v\left(I_{0}\right)\right) S^{n-1}+v^{\prime}\left(I_{0}\right) S^{n-1} \\
& \left.+\left(-M v^{0}+M v\left(I_{0}\right)+v^{\prime}\left(I_{0}\right)\right)\left(\sum_{i=1}^{n-1}(1-S) S^{i-1}-1\right)+v\left(I_{0}\right)(1-S) \sum_{i=1}^{n-1} i S^{i-1}\right\} \geq \Delta
\end{aligned}
$$

Using the fact that $\sum_{i=1}^{n-1} i S^{i-1}=\frac{1-S^{n}-n S^{n-1}(1-S)}{(1-S)^{2}}$, the above is simplified to be

$$
(\beta-\eta)\left\{\left(v^{c}-v\left(I_{0}\right)\right)\left(\frac{1-S^{n-1}}{1-S}\right)+S^{n-1} M\left(v\left(I_{0}\right)-v^{0}\right)\right\} \geq \Delta
$$

As prescribed in the review strategy, fix $M=\chi R, \chi>0$.

For large $n, S^{n-1} M \rightarrow 0$ and $\frac{1-S^{n-1}}{1-S} \rightarrow \frac{1}{1-S}$. Thus for all $\delta \in\left[\underline{\delta}^{\prime}, 1\right)$, there exists a $\underline{S}$ and a $\underline{R}^{\prime}$ such that for all $S>\underline{S}$ and $R>\underline{R}^{\prime}, \frac{\beta-\eta}{1-S}\left[v^{c}-v\left(I_{0}\right)\right]$ will exceed $\Delta$ for all $I_{R-n}=q-n$. Hence the customer's incentive constraint is violated and consequently fails the review only if $I_{t}<\max (0, t-(R-q))$.

Lemma 3 Let $I_{0}=0$. There exists a $\underline{\delta}^{\prime \prime}$ such that for all $\delta \in\left[\underline{\delta}^{\prime \prime}, 1\right)$, there exists a $\underline{R}^{\prime \prime}$ such that for $R>\underline{R}^{\prime \prime}$, the customer will truthfully share his private information and the supplier will respond to the customer's forecast for at least the first period of the review phase.

Proof. Consider the following sufficient condition for the customer's truth-telling constraint for hold: the passing of the review phase is deferred until $I_{t}$ is such that $I_{R-n} \geq q$. Because $v\left(I_{0}\right) \in\left[v^{0}, v^{c}\right]$, the truth-telling constraint will hold if the sufficient condition is imposed and the customer still forecasts truthfully.

Recall from claim proven above that the failing of the review occurs only when $I_{t}<\max (0, t-(R-q))$. Therefore, as $\delta \rightarrow 1$, if,

$$
(\beta-\eta)\left\{\begin{array}{c}
\left(v^{c}-v\left(I_{0}\right)\right)\left[\begin{array}{c}
q-2 \\
\sum_{i=0}\left(\begin{array}{c}
R-q+i \\
R-q
\end{array}\right) S^{i}(1-S)^{R-q}-\sum_{i=0}^{R-q-1}\left(\begin{array}{c}
q-1+i \\
q-1
\end{array}\right)(1-S)^{i} S^{q-1}
\end{array}\right] \\
+\left(\begin{array}{c}
R-1 \\
R-q
\end{array}\right) S^{q-1}(1-S)^{R-q} M\left(v\left(I_{0}\right)-v^{0}\right)
\end{array}\right\}
$$$$
>\Delta
$$ 
then the customer will truthfully share his private information at the start of the review phase.

The left-hand side of the inequality has three major components. The first one, $(\beta-\eta)\left(v^{c}-v\left(I_{0}\right)\right)$ $\sum_{i=0}^{q-2}\left(\begin{array}{c}R-q+i \\ R-q\end{array}\right) S^{i}(1-S)^{R-q}$, represents the gain from avoiding failing the review before period $R$. The review is terminated before period $R$ if the customer has had $R-q$ assessments where the trust index is not incremented. In this case, for each assessment where the trust index $i s$ incremented the customer avoids termination for an additional period up to the beginning of the final review period, i.e. $R-1$. The histories $S^{i}$ and $(1-S)^{R-q}$ can be arranged in $\left(\begin{array}{c}R-q+i \\ R-q\end{array}\right)$ combinations. Notice that for $i=q-2$, the summand becomes $\left(\begin{array}{l}R-2 \\ R-q\end{array}\right) S^{q-2}(1-S)^{R-q}$; this product multiplied by $(\beta-\eta)$ represents the probability of failing the review phase in period $R-1$.

Second, $(\beta-\eta)\left(v^{c}-v\left(I_{0}\right)\right) \sum_{i=0}^{R-q-1}\left(\begin{array}{c}q-1+i \\ q-1\end{array}\right)(1-S)^{i} S^{q-1}$ represents the marginal loss from passing the review before period $R$. The review is passed before period $R$ if the customer has had $q$ assessments where the trust index has been incremented before period $R$. If $I_{t}<q$, then the customer is assessed to have reported dishonestly and the passing of the review is delayed for an additional period. The passing of the review can be delayed for at most $R-q-1$ periods prior to the beginning of the final period. These histories $(1-S)^{i}$ and $S^{q-1}$ can be arranged in $\left(\begin{array}{c}q-1+i \\ q-1\end{array}\right)$ combinations.

And finally, $(\beta-\eta)\left(\begin{array}{l}R-1 \\ R-q\end{array}\right) S^{q-1}(1-S)^{R-q} M\left(v\left(I_{0}\right)-v^{0}\right)$ represents the marginal gain from avoiding the punishment phase in period $R$. This event occurs for a history of $R-q$ assessments where the trust index is not incremented and $q$ assessments where it is incremented. The histories $S^{q-1}$ and $(1-S)^{R-q}$ can be arranged in $\left(\begin{array}{l}R-1 \\ R-q\end{array}\right)$ combinations.

We now show that the constraint can always be satisfied if $R$ is sufficiently large and if $M=\chi R$, where $\chi>0$. Fix $M=\chi R$ and let $J=\tau R^{\rho}$ where $\tau>0, \frac{1}{2}<\rho<1$ for the remainder of the analysis. Recall that $q=R S-J$. Then let $R \rightarrow \infty$ and after applying Stirling's formula

$$
\lim _{R \rightarrow \infty} \frac{R !}{\sqrt{2 \pi} R^{\left(R+\frac{1}{2}\right)} e^{-n}}=1
$$

we get

$$
(\beta-\eta)\left(\begin{array}{l}
R-1 \\
R-q
\end{array}\right) S^{q-1}(1-S)^{R-q} M\left(v\left(I_{0}\right)-v^{0}\right)=\frac{\sqrt{R}(\beta-\eta) \chi\left(v\left(I_{0}\right)-v^{0}\right)}{\sqrt{2 \pi S(1-S)}} \rightarrow \infty .
$$


Similarly, it can be shown that $\left(\begin{array}{c}R-q+i \\ R-q\end{array}\right) S^{i}(1-S)^{R-q}<\left(\begin{array}{c}R-q+i+1 \\ R-q\end{array}\right) S^{i+1}(1-S)^{R-q}$ for all $i=$ $0,1,2, \ldots, q-3$, and also that, as $R \rightarrow \infty$,

$$
\left(\begin{array}{l}
R-2 \\
R-q
\end{array}\right) S^{q-2}(1-S)^{R-q}=\frac{R S-1}{S(R-1)} \frac{1}{\sqrt{2 \pi R S(1-S)}} \rightarrow 0
$$

Thus as $R \rightarrow \infty, \sum_{i=0}^{q-2}\left(\begin{array}{c}R-q+i \\ R-q\end{array}\right) S^{i}(1-S)^{R-q} \rightarrow 0$, and

$$
\sum_{i=0}^{R-q-1}\left(\begin{array}{c}
q-1+i \\
q-1
\end{array}\right)(1-S)^{i} S^{q-1} \rightarrow 0
$$

Since $\Delta$ is bounded and the inequality is strict, it follows that for $R>\underline{R}^{\prime \prime}$ and $\delta \in\left[\underline{\delta}^{\prime \prime}, 1\right)$, the customer will truthfully share his private information and the supplier will trust the customer's forecast for at least the first period of the review phase.

Lemma 4 The probability of failing the review when the players use the specified review strategies is less than or equal to the probability of failing the review when the customer reports in good faith and is evaluated at the end of period $R$ only and is said to fail the review if $I_{R}<q$ and pass the review if $I_{R} \geq q$.

Proof. Since once the customer passes the review, the review phase is terminated, it follows that the probability of failing the review under the specified review strategies (i.e. the customer can pass the review before the end of period $R$ and fail the review if for any $t<R, I_{t}<\max (0, t-(R-q))$; see claim 3.1) is less than or equal to the probability of failing the review if the customer can pass the review only at the end of period $R$ but can fail the review before period $R$ if for any $t<R, I_{t}<\max (0, t-(R-q))$. To see this, choose some history that results in the review been passed in the former review specification but failed in the latter specification.

If the customer reports demand in good faith then the probability of failing the review if the customer can pass the review only at the of period $R$ but can fail the review before period $R$ if for any $t<R$, $I_{t}<\max (0, t-(R-q))$ equals the probability of failing the review when the customer is evaluated at period $R$ only and is said to fail the review if $I_{R}<q$ and pass the review if $I_{R} \geq q$. To prove this consider: if a history occurs such that $I_{R}<q$, then it follows that there exists a history for the game such 
that $I_{t}<\max (0, t-(R-q))$. Conversely, if there exists a history such that $\left.I_{t}<\max (0, t-(R-q))\right)$, then $I_{t+(R-t)}<\gamma_{t}+(R-t)=q$. However, $I_{t+R-t} \leq I_{t}+(R-t)<q$. Thus $I_{R}<q$.

\section{Proofs of Theorem 2:}

First, we note neither the customer nor the supplier has an incentive to deviate. Given that the customer would share forecasts and order truthfully, the supplier's best response is to trust and build $K_{i}^{c}$. Therefore the supplier does not have a strict incentive to deviate from the characterized equilibrium strategies.

For the customer, the equilibrium is constructed so that if the truth-telling constraints are no longer satisfied, the review will be terminated and if $I_{t} \geq \gamma_{t}$, the customer passes the review, or if $I_{t}<\gamma_{t}$, the customer fails the review. Thus the customer follows a sequentially rational strategy and has no incentive to deviate.

Next we show that under the specified strategy, the customer would almost always share forecasts and place orders truthfully. This is done with the following steps:

(1) The customer will only fail the review when $I_{t}<\max (0, t-(R-q))$,i.e., when $I_{t}$ is such that the customer will fail the review for sure. A sufficient condition of the above is to show that the customer has an incentive to share forecasts truthfully when $I_{R-n}=q-n$, which we have proven in Lemma 2 .

(2) From Lemma 3, there exist parameters of the game such that the customer will share forecasts truthfully at the beginning of the game.

(3) We now show that the characterized equilibrium yields almost full revelation of the customer's private information. Since the review phase may terminate earlier than at the end of period $R$, the following sufficient conditions are imposed to obtain a lower bound on the customer's normalized discounted expected profit: assume that during the review phase the customer reports in good faith but if he fails, then he fails the review at the earliest possible period, i.e. $t=R-q$; and assume that the customer reports in good faith and if he passes, then he passes the review at the earliest possible time period denoted as $t^{\prime}$; recall that $v\left(I_{0}\right) \in\left[v^{0}, v^{c}\right]$ and $t^{\prime}>0$ (as shown in claim 3 above). The Markovian Property of this repeated game yields a lower bound on the customer's normalized discounted expected profit, $v\left(I_{0}\right)$ : 


$$
\begin{aligned}
v\left(I_{0}\right) & \geq \lambda\left[(1-\delta) \sum_{i=1}^{R-q} \delta^{i-1} v^{c}+\delta^{R-q}\left(1-\delta^{M}\right) v^{0}+\delta^{R-q+M} v\left(I_{0}\right)\right] \\
& +(1-\lambda)\left[(1-\delta) \sum_{i=1}^{t^{\prime}} \delta^{i-1} v^{c}+\delta^{t^{\prime}} v\left(I_{0}\right)\right] .
\end{aligned}
$$

Solving for $v\left(I_{0}\right)$ yields:

$$
v\left(I_{0}\right) \geq \frac{\left(1-\delta^{R-q}\right) v^{c} \lambda+\delta^{R-q}\left(1-\delta^{M}\right) v^{0} \lambda+\left(1-\delta^{t^{\prime}}\right) v^{c}(1-\lambda)}{1-\delta^{R-q+M} \lambda-\delta^{t^{\prime}}(1-\lambda)} .
$$

Substitute for $M=\chi R$ and observe that $\lim _{R \rightarrow \infty} \frac{M}{R-q}=\frac{\chi}{1-S}$. Since $t^{\prime}>0$, it follows that the left-hand side of the inequality in expression (11) converges uniformly in $R$ as $\delta$ approaches 1 to

$$
\frac{\left(v^{c}+\frac{M}{R-q} v^{0}\right) \lambda+\frac{t^{\prime}}{R-q} v^{c}(1-\lambda)}{\frac{R-q+M}{R-q} \lambda+\frac{t^{\prime}}{R-q}(1-\lambda)} .
$$

Lemma 4 shows that the probability of failing the review when the players use the specified review strategies is less than or equal to the probability of failing the review when the customer reports in good faith and is evaluated at period $R$ only and is said to fail the review if $I_{R}<q$ and pass the review if $I_{R} \geq q$; this upper bound is denoted $\bar{\lambda}\left(I_{R}<R . S-J\right)$. If the customer reports in good faith, then $I_{R}$ is a binomial random variable. Invoking Chebychev's inequality implies that $\bar{\lambda}\left(I_{R}<R . S-J\right) \leq \frac{R S(1-S)}{J^{2}}$. If $J=\beta R^{\rho}$, where $\beta>0$ and $\frac{1}{2}<\rho<1$, then $\bar{\lambda}\left(I_{R}<R . S-J\right)$ approaches zero as $R$ increases with out limit; since $\lambda$ is bounded from above by $\bar{\lambda}\left(I_{R}<R . S-J\right), \lambda \rightarrow 0$.

Hence, for any $\varepsilon>0$, there exists a discount factor $\underline{\delta}$ such that for all $\delta \in[\underline{\delta}, 1)$, there exists a $\underline{R}$ such that for all $R>\underline{R}, V\left(I_{0}\right)>v^{c}-\varepsilon$. This provides a lower bound on the customer's expected profits. Moreover, $\lambda$ can be made arbitrarily close to zero by choosing $R$ sufficiently large. 


\section{References}

[1] Abreu, D. (1988). "On the Theory of Infinitely Repeated Games with Discounting." Econometrica 56(2): 383-396.

[2] Arrow, K. J., "The Economics of Agency," in J.W. Pratt and R. J. Zeckhauser (Eds.), Principals and Agents, Harvard Business School Press, Cambridge, MA, 1985.

[3] Bajari, P. and S. Tadelis (2001). "Incentives versus transaction costs: A theory of procurement contracts." The Rand Journal of Economics 32(3): 387.

[4] Baker, G., R. Gibbons and K. J. Murphy (2002). "Relational contracts and the theory of the firm." Quarterly Journal of Economics 117(1): 39-84.

[5] Barnes-Schuster, D., Y. Bassok and R. Anupindi (2002). "Coordination and flexibility in supply contracts with options." Manufacturing \& Service Operations Management 4(3): 171.

[6] Beth, S., D. N. Burt, W. Copacino, C. Gopal, H. L. Lee, R. P. Lynch and S. Morris (2003). "Supply chain challenges - Building relationships." Harvard Business Review 81(7): 64-+.

[7] Cachon, G. P. and M. A. Lariviere (2001). "Contracting to assure supply: How to share demand forecasts in a supply chain." Management Science 47(5): 629-646.

[8] Cachon, G. P. and M. A. Lariviere (2005). "Supply Chain Coordination with Revenue-Sharing Contracts: Strengths and Limitations." Management Science 51(1): 30.

[9] Chen, F. (2002). Information Sharing and Supply Chain Coordination. Handbook of Operations Research and Management Science: Supply Chain Management. T. d. Kok and S. Graves, North-Holland.

[10] Chen, F. (2005). "Salesforce incentives, market information, and production/inventory planning." Management Science 51(1): 60-75.

[11] Cohen, M. A., T. H. Ho, Z. J. Ren and C. Terwiesch (2003). "Measuring Imputed Cost in the Semiconductor Equipment Supply Chain.” Management Science 49(12): 1653. 
[12] Corbett, C. J. (2001). "Stochastic inventory systems in a supply chain with asymmetric information: Cycle stocks, safety stocks, and consignment stock." Operations Research 49(4): 487-500.

[13] Corbett, C. J., D. Zhou and C. S. Tang (2004). "Designing Supply Contracts: Contract Type and Information Asymmetry." Management Science 50(4): 550.

[14] Crawford, V. P. and J. Sobel (1982). "Strategic Information-Transmission." Econometrica 50(6): 14311451.

[15] Desai, P. S. and K. Srinivasan (1995). "Demand signalling under unobservable effort in franchising: Linear and nonlinear price contracts." Management Science 41(10): 1608.

[16] Farlow, D., G. Schmidt and A. Tsay (1996). Supplier management at Sun Microsystems (A), Stanford Graduate School Business.

[17] Friedman, J. W. (1971). "Non-Cooperative Equilibrium for Supergames." Review of Economic Studies 38(113): 1-12.

[18] Friedman, J. W. (1986). Game theory with applications to economics. New York, Oxford University Press.

[19] Fujimoto, T. (1999). The evolution of a manufacturing system at Toyota. New York, Oxford University Press.

[20] Gibbons, R. (1992). Game theory for applied economists. Princeton, N.J., Princeton University Press.

[21] Ha, A. Y. (2001). "Supplier-buyer contracting: Asymmetric cost information and cutoff level policy for buyer participation." Naval Research Logistics 48(1): 41-64.

[22] Hagen, J. M. and S. Choe (1998). "Trust in Japanese interfirm relations: Institutional sanctions matter." Academy of Management. The Academy of Management Review 23(3): 589.

[23] Iyer, G. and J. M. Villas-Boas (2003). "A Bargaining Theory of Distribution Channels." Journal of Marketing Research 40(1): 80. 
[24] Kulp, S. C., E. Ofek and J. Whitake (2003). Supply-Chain Coordination: How Companies Leverage Information Flows to Generate Value. The practice of supply chain management. C. Billington, H. Lee, J. Neale and T. Harrison, Kluwer Publishing.

[25] Lariviere, M. A. and V. Padmanabhan (1997). "Slotting allowances and new product introductions." Marketing Science 16(2): 112-128.

[26] Levin, J. (2003). "Relational incentive contracts." The American Economic Review 93(3): 835.

[27] Liker, J. K. (2004). The Toyota Way: 14 Management Principles from the World's Greatest Manufacturer, McGraw-Hill.

[28] McMillan, J. (1990). "Managing Suppliers - Incentive Systems in Japanese and United-States Industry." California Management Review 32(4): 38-55.

[29] Minahan, T. (1998a). "Purchasing needs to do more than measure." Purchasing 124(1): 59.

[30] Minahan, T. (1998b). "Is partnering a sham?" Purchasing 124(2): 61.

[31] Morris, S. (2001). "Political correctness.” Journal of Political Economy 109(2): 231-265.

[32] Ozer, O. and W. Wei (2006). "Strategic commitment for optimal capacity decision under asymmetric forecast information." Management Science 52(8): 1238-1257.

[33] Pasternack, B. A. (1985). "Optimal Pricing and Return Policies for Perishable Commodities." Marketing Science 4(2): 166 .

[34] Plambeck, E. L. and T. A. Taylor (2006). "Partnership in a Dynamic Production System with Unobservable Actions and Noncontractible Output." Management Science 52(10): 1509-1527.

[35] Porteus, E. L. and S. Whang (1991). “On Manufacturing Marketing Incentives." Management Science 37(9): 1166-1181. 
[36] Pyke, D. F. and E. M. Johnson (2003). Sourcing strategies and supplier relationships: alliances vs. eProcurement. The practice of supply chain management. C. Billington, H. Lee, J. Neale and T. Harrison, Kluwer Publishing: 77-89.

[37] Radner, R. (1985). "Repeated Principal-Agent Games with Discounting." Econometrica 53(5): 11731198.

[38] Spengler, J. J. (1950). "Vertical Integration and Anti-Trust Policy." Journal of Political Economy 58: $347-352$.

[39] Stocken, P. C. (2000). "Credibility of voluntary disclosure." Rand Journal of Economics 31(2): 359-374.

[40] Taylor, T. A. (2002). "Supply chain coordination under channel rebates with sales effort effects." Management Science 48(8): 992.

[41] Taylor, T. A. and E. L. Plambeck (2006). "Supply Chain Relationships and Contracts: the Impact of Repeated Interaction on Capacity Investment and Procurement." Management Science, forthcoming..

[42] Taylor, T. A. and E. L. Plambeck (2007). "Simple Relational Contracts for Capacity Investment: Priceonly versus Quantity Commitment." Manufacturing and Service Operations Management 9(1): 94-113.

[43] Terwiesch, C., Z. J. Ren, T. H. Ho and M. A. Cohen (2005). "An empirical analysis of forecast sharing in the semiconductor equipment supply chain.” Management Science 51(2): 208-220.

[44] Tomlin, B. (2003). "Capacity Investments in Supply Chains: Sharing the Gain Rather Than Sharing the Pain.” Manufacturing \& Service Operations Management 5(4): 317.

[45] Tsay, A. A. (1999). "The quantity flexibility contract and supplier-customer incentives." Management Science 45(10): 1339 .

[46] Van Mieghem, J. A. (1999). "Coordinating investment, production, and subcontracting." Management Science 45(7): 954-971. 